\title{
Complications and Carcinogenic Effects of Mustard Gas - a Systematic Review and Meta-Analysis in Iran
}

\author{
Yunes Panahi ${ }^{1}$, Nasrin Gholami², Morteza Ghojazadeh ${ }^{3}$, Farnaz Moslemi ${ }^{4}$, \\ Mohammad Naghavi-Behzad $^{5}$, Saber Azami-Aghdash ${ }^{6}$,Alireza Ghaffari ${ }^{7 *}$, Reza \\ Piri $^{7 *}$
}

\begin{abstract}
Background: Catastrophic effects of mustard gas as a chemical warfare agent have always been a major problem for those exposed to this agent. In this meta-analysis it was tried to evaluate carcinogenesis, ocular, cutaneous and respiratory complications of mustard gas exposure among Iranians who had been exposed to this agent during the Iran-Iraq war. Materials and Methods: In this meta-analysis, the required data were collected using keywords "mustard gas", "sulfur mustard", "cancer", "neoplasm", "respiratory complications", "ocular complications", "lung disease", "chronic complication", "eye", "skin”, "cutaneous complication", "carcinogenesis" and their combination with keywords "Iran", "Iranian", "prevalence", "mortality" and their Farsi equivalent terms from the databases of SID, Iranmedex, Magiran, Pubmed, Science Direct, Google Search engine, Gray Literature and Reference of References. To determine the prevalence of each complication and perform meta-analysis, CMA: 2 (Comprehensive Meta-Analysis) software with a randomized model was used. Results: Of the 542 articles found, 7 national articles, consistent with the aims of this study were selected. Metaanalysis of seven papers revealed that cancer risk, especially cancer of the respiratory system was elevated, so that the relative risk (RR) of cancer role of mustard gas was inconsistent from $2 / 1$ to 4 in this survey. Also prevalence of delayed skin disorders due to sulfur mustard was $94.6 \%$, pulmonary complications $94.5 \%$ and ocular complications $89.9 \%$. The incidence of various cancers in victims exposed to mustard gas was $1.7 \%$ worldwide where the rate was $2.2 \%$ in Iranian victims of the Iraq-Iran war. Conclusions: Based on present study the prevalence of delayed mustard gas related cutaneous, pulmonary and ocular complications is above $90 \%$ and risk of carcinogenesis is higher in comparison to worldwide statistics. This may suggest need for long-term and persistent follow-up and rehabilitation procedures for populations exposed to this agent.
\end{abstract}

Keywords: Mustard gas - carcinogenic - neoplasm - complication - Iran - meta-analysis

Asian Pac J Cancer Prev, 16 (17), 7567-7573

\section{Introduction}

Sulfur mustard the common name given to 1,1-thiobis(2-chloroethane) is a well-known chemical warfare agent which causes blistering of the mucous membranes and skin on contact. Sulfur Mustard also known as Mustard Gas (MG) is a chemical warfare agent which is an odourless and colourless oily liquid and has a high stability in the environment (Security, 2011). MG is divided into two kinds: nitrogen mustard, which is used for treatment of cancer, and sulfur mustard, which is used as a poison gas in the wars (Marzban, 1986).

This compound eliminates a chloride ion by intramolecular nucleophilic substitution to produce a cyclic sulfonium ion which is a reactive intermediate tending to cause permanent alkylation of the guanine nucleotide in DNA, which prevents cellular division and generally leads directly to apoptosis or, if cell death is not immediate, this damage may lead to a malignant transformation (Safarinejad, 2001; McClintock et al., 2002). Historically MG was used for treatment of psoriasis (Le and Knudsen, 2006; Barar and Omidi, 2012; Ghabili et al., 2013; Sokouti et al., 2013). But today it has no medical usage. This chemical weapon was again used in the Iraq-Iran war in years of 1980-1988 which extensively targeted unprotected civilians in Iran and within the Kurdish regions of Iraq (Emad and Rezaian, 1997). MG exposure usually is not fatal and also exposed individuals may not know immediately that they have been exposed, because MG may not have an odour or have an odour that might

${ }^{I}$ Chemical Injuries Research Center, Baqiyatallah University of Medical Sciences, ${ }^{2}$ Hematology and Oncology Research Center, ${ }^{3}$ Liver and Gastrointestinal Disease Research Center, ${ }^{4}$ Women's Reproductive Health Research Center, ${ }^{5}$ Students' Research Committee, ${ }^{7}$ Medical Philosophy and History Research Center, Tabriz University Medical Sciences, ${ }^{6}$ Health Management and Economics Research Center, Iran University of Medical Sciences, Tabriz, Iran*For correspondence: agh1343@gmail.com,dr.reza.piri@gmail.com 
warn. Typically, signs and symptoms do not happen right away and depend on the severity of the exposure, symptoms and signs may not occur for up to 24 hours, which includes intense itching second- and third degree burns and skin irritation, which progressively turns into large blisters, which are very debilitating (Stuart et al., 2003; Kehe and Szinicz, 2005; Mirsadraee et al., 2005; Naraghi et al., 2005; Jabbari et al., 2012; Saleh et al., 2013; Mortazavi et al., 2014). Moreover many studies declared that this poison gas is carcinogenic both in animals and humans (Zandieh et al., 1990; Eisenmenger et al., 1991; Program, 2011).

In a study investigating patients exposed to chemical warfare 25 years after wartime, c-DNA microarray profiling indicated that a total of specific 122 genes were significantly transformed in the lungs and airways of MG exposure victims (Bhavana et al., 2014; Najafi et al., 2014). Those genes all take part in functions commonly affected by MG exposure, including inflammation and apoptosis (Chatterjee et al., 2003; Ray et al., 2008).

In another study investigating late cutaneous manifestations 14 to 20 years after wartime exposure to MG, it was concluded that Skin lesions are seen in more than $90 \%$ of the patients exposed to MG (Emadi et al., 2008; Amirnia et al.,2012; Gharemohammadlou et al., 2013).

In other studies conducted in Iran, varied prevalence for cancer and delayed complications has been reported (Marzban, 1986; Emad and Rezaian, 1997; Agin and Broumand, 2004; Riazi et al., 2004; GhasemiBroumand et al., 2006; Gilasi et al., 2006; GhasemiBroumand et al., 2007; GhasemiBroumand and Amiri, 2008; Namazi et al., 2009; Ghabili et al., 2013). Despite the announcement of different figures and statistics for carcinogenesis, respiratory, cutaneous and ocular complications, literature review indicates that no appropriate conclusion has been achieved from these studies. Therefore, the present meta-analysis aimed at assessing the studies conducted in this field to achieve a solid conclusion derived from evaluation of carcinogenesis, respiratory, cutaneous and ocular complications reported from Iran 25 years after the last MG utilization by Iraqi military forces against Iranian soldiers and civilians in Iraq-Iran war 1980-1988.

\section{Materials and Methods}

This Meta-analytical study is based on all results obtained from studies meeting inclusion criteria. Data were collected by using keywords "mustard gas", "sulfur mustard", "cancer", "neoplasm", "respiratory complications", "ocular complications", "lung disease", "chronic complication", "eye", "skin", "cutaneous complication", "carcinogenesis" and their combination with keywords "Iran", "Iranian", ,prevalence", "mortality" and their Farsi equivalent terms from the databases of SID, Iranmedex, Magiran, Pubmed, Science Direct, Google Search engine, Gray Literature and Reference of References. Considering the cessation of hostilities between Iraq and Iran in 1988 and investigating MG exposure complications as the objective of this study, a time span of 1995 to 2013 was selected. Both
Persian and English published articles were searched and reviewed. Inclusion criteria for the study included studies focusing on carcinogenic, respiratory, cutaneous and ocular complications of MG exposure in Iranian population and highlighting the carcinogenic effects and delayed complications of this warfare agent. Exclusion criteria from the study included the letter to editorials, proceedings papers, case reports, interventional, quality of life, psychological, immunological, diagnostic and animal and incidence studies. Furthermore studies on the similar complications of mustard gas and other gases were excluded. Then two evaluators assessed the quality of the selected articles using designated checklists. Cases on which the two evaluators did not agree were discussed first if no conclusion was drawn it was referred to the third evaluator. Firstly, the titles of all articles were assessed and those not consistent with the objectives of the study were excluded. Later, abstract and the main text of the articles were evaluated respectively to identify studies meeting exclusion criteria and those with weak correlation with the objectives of our study. The initial search resulted in 542 articles. After elimination of the unrelated, poorly related and similar articles, seven articles were included. Selected papers were entirely reviewed. Figure 1 illustrates inclusion and exclusion procedure of the articles.

\section{Statistics}

To evaluate the carcinogenic effects and prevalence of each complication and to perform meta-analysis, CMA (Comprehensive Meta-Analysis) software version 2 (Biostat, Englewood, NJ) with confidence interval of 95\% was used. Forest plots were used to show effect size and report the results. To check the status of articles entered into the study in terms of publication bias funnel diagram was used and the statistical test of Egger was used to assess the publication bias. P value less than $0.05 \%$ was considered statistically significant in this study.

\section{Results}

Of 542 initially included articles, at last seven articles were included. The characteristics of the chosen articles in this study are presented in extraction table (Table 1).

Randomization model in meta-analysis was used based on the result of Cochrane Q test indicating heterogeneity between the results of different studies. Chi-square test was used to calculate the differences between frequencies.

Of all studies, 4962 patients who had been exposed

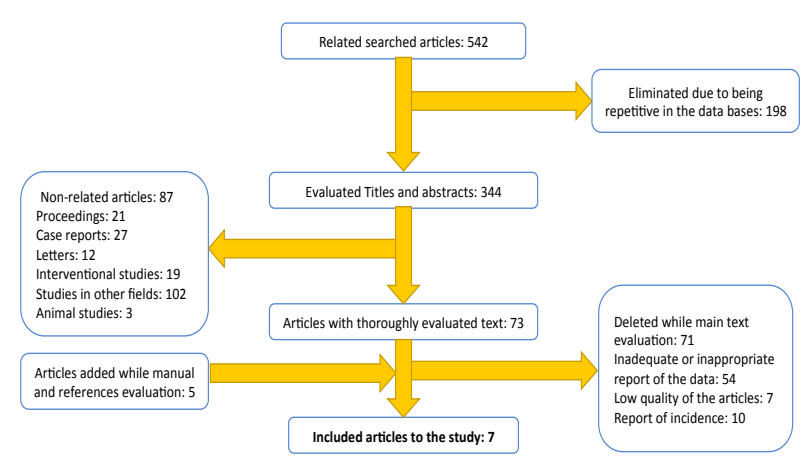

Figure 1. Process of Selecting the Articles 

Table 1. Characteristics of the Assessed Articles ( $n=7)$

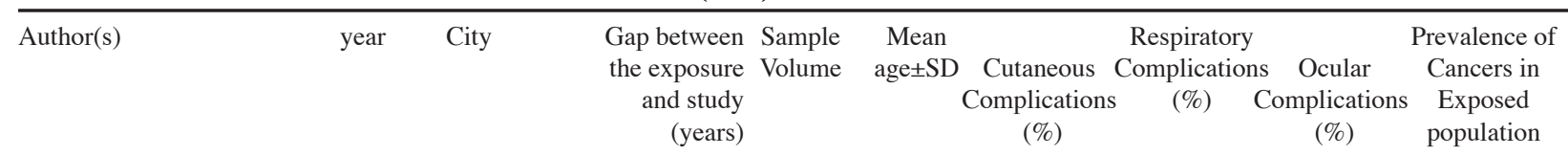

\begin{tabular}{|c|c|c|c|c|c|c|c|c|c|}
\hline & & & & & & & & & $(\%)$ \\
\hline Namazi et al. & 2009 & Shiraz & -22 & 134 & $37.2 \pm 9$ & 82.8 & 100 & 77.6 & 1.5 \\
\hline GhasemiBroumand et al. & 2006 & Sardasht & 17 & 600 & $41.03 \pm 14.3$ & 83.2 & 81.7 & 69.2 & - \\
\hline GhasemiBroumand et al. & 2008 & Iran-BonyadeShohada & 10 & 500 & $5.2 \pm 36.5$ & 99.2 & 100 & 100 & 2 \\
\hline Riazi et al. & 2008 & Different Iranian cities & - & 2252 & $34.31 \pm 9.05$ & - & - & 89.8 & - \\
\hline Agin et al. & 2004 & Different Iranian cities & 15 & 500 & $5.2 \pm 36.5$ & 99.2 & 69.2 & 80 & - \\
\hline GhasemiBroumand et al. & 2007 & Marivan 17 & -22 & 476 & - & 90.8 & 96.6 & 89.2 & 0.75 \\
\hline Ghilasi et al. & 2006 & Isfahan & \pm 13 & 500 & $41 \pm 10$ & - & 96.3 & 71.2 & 3 \\
\hline
\end{tabular}

Study name

Event rate

And $95 \% \mathrm{Cl}$

Ghasemi Broumand et al; 2008

Namazi et al; 2006

Ghasemi Broumand et al; 2005

Ghasemi Broumand et al; 2002

Agin and Ghasemi; 2002

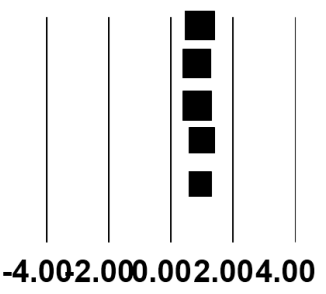

Figure 2. The Prevalence of Delayed Cutaneous Mustard Gas Related Complications in the Victims of Chemical Warfare Agents in Iraq-Iran war with a Confidence interval of $95 \%\left(I^{2}=95\right.$ Based on Randomized Model)

\begin{tabular}{ll}
\hline Study name & Event rate \\
& And $95 \% \mathrm{Cl}$
\end{tabular}

Namazi et al; 2006

Ghasemi Broumand et al; 2005

Ghasemi Broumand et al; 2002

Ghilasi et al; 2006

Agin and Ghasemi; 2002

Ghasemi Broumand et al; 2008

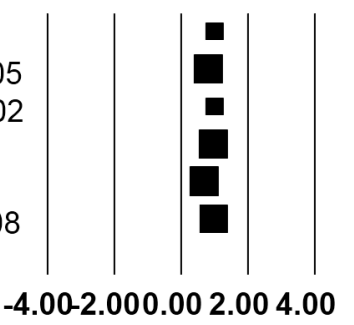

Figure 3. The Prevalence of Delayed Respiratory Mustard Gas Related Complications in the Victims of Chemical Warfare agents in Iraq-Iran war with a Confidence Interval of $95 \%\left(I^{2}=97.2\right.$ Based on Randomized Model)

to MG was included in this Meta-analysis. All included articles were reported on an average of $18 \pm 6.4$ years after the MG exposure.

In this study, the prevalence of delayed cutaneous MG related complications in the victims of chemical warfare agents in Iraq-Iran war was 94.6\% (Figure 2, 88.3-97.7 percent, 95\% CI) (Q value $=80.21, \mathrm{df}=4, \mathrm{P}$ value $<0.001)$.

The prevalence of delayed respiratory MG related complications in the victims of chemical warfare agents in Iraq-Iran war was $94.5 \%$ (Figure 3,86.4-97.7 percent, $95 \% \mathrm{CI})(\mathrm{Q}$ value $=181.70, \mathrm{df}=5, \mathrm{P}$ value $<0.001)$.

The prevalence of delayed ocular MG related complications in the victims of chemical warfare agents in Iraq-Iran war was $89.9 \%$ (Figure 4, 83.7-93.9 percent, $95 \% \mathrm{CI})(\mathrm{Q}$ value $=95.6, \mathrm{df}=5, \mathrm{P}$ value $<0.001)$
Study name

Event rate

And $95 \% \mathrm{Cl}$
Namazi et al; 2006

Ghasemi Broumand et al; 2005

Ghasemi Broumand et al; 2002

Riazi et al; 2005

Agin and Ghasemi; 2002

Ghasemi Broumand et al; 2008

Ghilasi et al; 2006

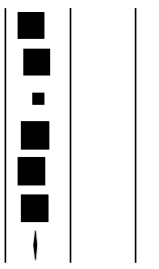

$-4.00-2.000 .002 .004 .00$

Figure 4. The Prevalence of Delayed Ocular Mustard Gas Related Complications in the Victims of Chemical Warfare agents between Iraq and Iran with a Confidence interval of $95 \%\left(I^{2}=94.7\right.$ Based on Randomized Model)

Study name

Event rate And $95 \% \mathrm{Cl}$

Namazi et al; 2006

Ghasemi Broumand et al; 2005 Ghasemi Broumand et al; 2002 Riazi et al; 2005

Agin and Ghasemi; 2002

Gillassi et al

Soroush MR et al; 2009

Geraci MJ et al; 2008

Ghanei M et al; 2007

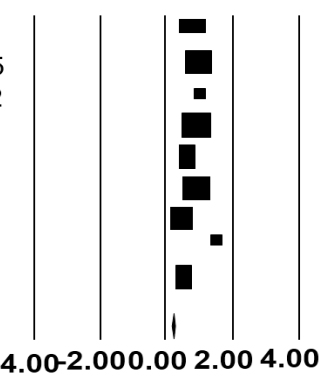

Figure 5. The Incidence of Different Types of Cancers Due to Mustard Gas in the Victims of this agents Worldwide with a Confidence Interval of $95 \%\left(I^{2}=88.2\right.$ Based on Randomized Model)

Study name

Event rate And 95\% CI

Namazi et al; 2006

Ghasemi Broumand et al; 2005 Ghasemi Broumand et al; 2002 Riazi et al; 2005

Agin and Ghasemi; 2002

Soroush MR et al; 2009

Ghanei M et al; 2007

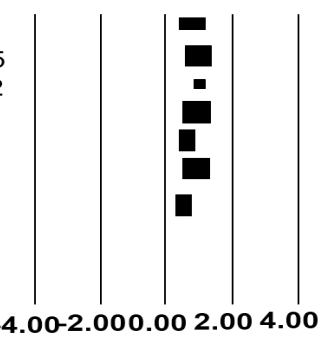

Figure 6. The Incidence of Different Types of Cancers Due to Mustard Gas in the Chemically Victims of IraqIran war with a Confidence Interval of $95 \%\left(I^{2}=92.7\right.$ Based on Randomized Model) 


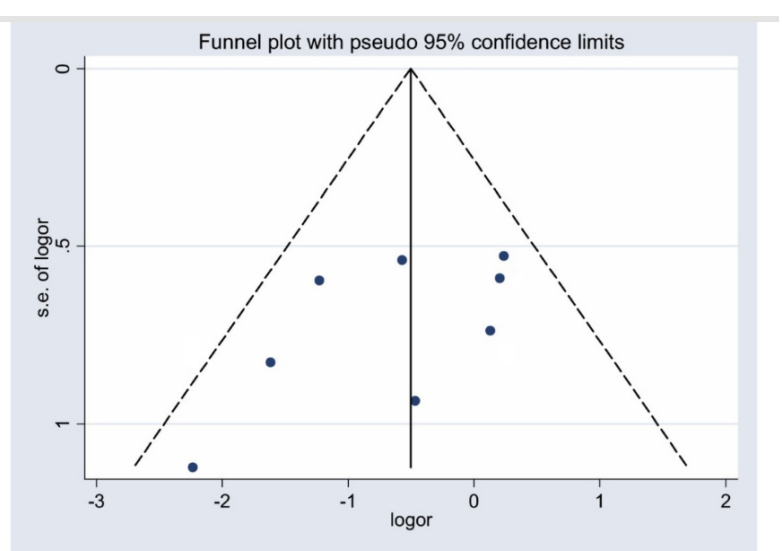

Figure 6. Assessment of Publication Bias of Analysed Articles Based on Funnel Plot

Table 2. Egger's Test for Publication Bias

\begin{tabular}{lccc}
\hline & Coefficient & Standard Error & P value \\
\hline Slop & 1.58 & 1.02 & 0.26 \\
Bias & -2.21 & 7.68 & 0.38 \\
\hline
\end{tabular}

The incidence of different types of cancers due to MG exposure in the victims of this agents worldwide was $1.7 \%$ based on randomized model (Figure 5, 1.1- 2.9, 95\% CI) $(\mathrm{Q}$ value $=104.4, \mathrm{df}=8, \mathrm{P}$ value $<0.001)$.

Then the prevalence of cancers in Iranian victims of this chemical warfare in Iraq-Iran war was $2.2 \%$ based on randomized model with a confidence interval of $95 \%$ (Figure 6 1.03-3.4 percent, 95\% CI) (Q value $=15.1, \mathrm{df}=6$, $\mathrm{P}$ value $<0.001)$. Of all included patients lung neoplasm were the most common type neoplasms (53.21 percent) after gastrointestinal neoplasms (41.45 percent).

According to forest plot, relative risk estimates of rapid and acute $\mathrm{MG}$ exposure role in incidence of various cancers was variable from 2.1 to 4 in all selected studies, while this incidence higher in Ghilasi et al. (Gilasi et al., 2006) Study than other studies.

To determine the presence of publication bias, the Egger's Statistical test was used (Table 2). Also publication bias is shown based on funnel plot in Figure 7.

Lack of congruence among included studies was mostly due to epidemiological and methodological erorrs in these studies.

\section{Discussion}

MG as a potent chemical warfare agent which was first synthesized in 1822, but had not been used as a warfare agent till 1860, when its harmful effects were discovered. MG is a DNA-alkylating agent and extremely cytotoxic at low concentrations and causes gene mutations in a dosedependent fashion. Its uses in wars were responsible for a wide variety of adverse health effects, such as lethality, vesication, erythema, ocular and pulmonary effects and burns on the skin (Karalliedde et al., 2000). In present study cutaneous, ocular and respiratory complications of MG exposure were all more than $90 \%$ and prevalence of cancers among those exposed to $\mathrm{MG}$ was $2.2 \%$.

Exposure to MG (military or occupational) is associated with an increased risk of lung or other respiratory-tract cancers in humans (Ghabili et al., 2010;
Doi et al., 2011; Zafarghandi et al., 2013). A cohort study in England found significant excesses of laryngeal, pharyngeal, upper-respiratory-tract, and lung cancer in workers of a MG manufacturing centers during World War II (Easton et al., 1988). Also several studies have been shown that injection or inhalation of MG caused lung tumors in rodent (Heston, 1953; Sasser et al., 1996). This was similar to finding of present study.

The carcinogenic potential of MG is clearly established in humans and experimental animals. Numerous evidences of carcinogenesis of MG among humans are based on data sets obtained from workers of MG manufacturing centers who were involved with the production of MG, so they were continuously exposed to MG. The studies of acute exposure, resulting from use of the MG in World Wars and during the Iraq-Iran war, have resulted in different findings.

The theory of considering MG as human carcinogen was supported primarily by repeated reports of association between occupational MG exposure and lung cancer in Japanese and English MG manufacturing workers (Shigenobu, 1980; Manning et al., 1981; Nishimoto et al., 1983). In 1968, Wada et al (Wada et al., 1968) reported that 33 of the workers who worked at MG manufacturing centers died from respiratory system cancers. Further support for the carcinogenic effect of MG is provided by studies which shown lung cancer in factory workers results after the accumulation of 6 mutations (Doi et al., 2011); carcinogenesis process is a multistep occurrence initiating with hyperplasia, moving through metaplasia, dysplasia, and carcinoma, and in the end becoming invasive neoplasm (Tokuoka et al., 1986). Other studies provide epidemiological evidence, during World Wars I and II, that young male soldiers who were exposed to MG in combat had slight increased incidences of lung cancer deaths, however these studies were carried out years after wartime use and only after the dreadful use of MG by Iraqi forces as a battlefield force multiplier upon unprotected civilian populations in Iran(Abbas, 1984) and multiple accidental and environmental exposures (Le and Knudsen, 2006; Geraci, 2008), long-term studies have been conducted on exposed civilians, including women and children.

A long-term follow-up (25 years) cohort study was conducted among 7,570 among Iranian veterans exposed to MG and equivalent matched to exposed group in each (Zafarghandi et al., 2013) Types of cancers reported in this study included gastrointestinal, lung and bronchial, lymphatic and hematology, genitourinary, skin, head and neck, CNS, bone and soft tissue and others. In this report, the relative risk of malignant disorder occurrence for MG exposure was 2.02 and the incidence rate of cancer in MG exposed veterans was nearly two fold greater than the unexposed subjects in non-smokers participants. Although incidence rate of cancer was significantly different between overall ages, the authors indicated that no statistically significant differences were obvious for any type of cancer; Although in contrast to this study, present study indicated that most common type of cancer among MG exposed victims are squamous cell carcinoma of lung, but relative risk attributed to $\mathrm{MG}$ exposure for 
cancer development is similar to what this study presents.

In a survey of 289 Iranian veterans who were exposed to high doses of MG between 1983 and 1989 during the Iran-Iraq war, none of the veterans had experienced urogenital malignancies (Soroush et al., 2009). However, it has been previously reported high levels of toxin might be excreted into urine in acute phase of MG exposure. (GHANEEI et al., 2007). The authors indicated that no malignancy screening was used in this study so the absence of urogenital neoplasms in the studied sample remained unidentified. Another possible scenario explaining this issue might be the shorter development period of common neoplasm related to MG exposure in comparison to urogenital malignancies.

A two-year prospective study was conducted on 43 male Iranian veterans who suffered MG exposure (Zojaji et al., 2009). Of seven patients with mostly head and neck problems, two patients had thyroid cancer. The first with undifferentiated thyroid carcinoma and the second with papillary carcinoma of a thyroglossal cyst which have been diagnosed 12 and 14 years post exposure, respectively. Also one patient had nasopharyngeal carcinoma, 12 years after MG exposure; in contrast, in present study most of the cancers were related to lower respiratory tract.

To assess the increased risk of chronic myelocytic leukemia occurrence, 665 war victims exposed to MG during the Iraq-Iran war from 1983 to 1989 were studied(Ghanei and Vosoghi, 2002). Two cases with CML in 2 consecutive years were found and this means the occurrence rate of 1 case per 2,500. The calculated prevalence is 400 times greater than that in the normal population (about 1 per 100,000). The authors indicated that they could not ascertain whether combined chemical gases were used along with MG in the study group.

Emad et al. (Emad and Rezaian, 1997) reported that acute and massive inhalation of MG by a group of Iranian veterans in 1986 did not produce bronchial carcinoma or other lung malignancies in these veterans after 10 years, although lung cancer has been reported as a late complication of MG inhalation among factory workers who manufactured MG (Wada et al., 1968; Fraumeni Jr, 1975; Easton et al., 1988). Another study of 500 chemically-injured soldiers with at least one episode of exposure to MG (Gilasi et al., 2006) showed no statistically significant relationship between cancer incidence and acute exposure to MG 18 years post exposure.

A study conducted to find out whether a single exposure to MG with respiratory injury was associated with increased risk of lung cancer in later life including 7,151 U.S. white males from 1956 to 1965 concluded that carcinogenic effect of MG depends on the duration of exposure and there was increased risk with increased duration of exposure, in no case the level or duration of exposure was quantified (Norman, 1975).

In a study investigating long-term complications of MG exposure in severely intoxicated Iranian veterans, it was concluded that The most common complications were related to the lungs, peripheral nerves, skin, and eyes .(95\%, $77.5 \%, 75 \%$ and $65 \%$, respectively)(Balali-Mood et al., 2005) although in present study peripheral nerve complications were not studied, but just like this study respiratory complications are of great prevalence $(94.5 \%)$ , but ocular and cutaneous complications in present study are more prevalent.

In a study assessing long-term respiratory complications veterans exposed to $\mathrm{MG}$, it was concluded that the cumulative prevalence rate of complications related to lung was about 30 percent and the first year of follow-up had the lowest annual incidence rate (Zarchi et al., 2004); this study reports a prevalence less than what is reported in present study, this difference might be due to the definition of respiratory complications mentioned in this study.

Late cutaneous complications of MG exposure 14 to 20 years after wartime in a study concluded that nonspecific cutaneous manifestations were about 90 percent present among those exposed to MG; this manifestations included eczema, dermatitis, multiple angiomas (and several other conditions. The relationship between frequency of eczema, dermatitis, tinea versicolor, vitiligo, and urticaria among patients exposed to GM was statistically significant (Emadi et al., 2008) this was similar to what the present study has concluded.

Although the present study provides a systematic meta-analysis for the prevalence of MG related delayed complications, it is also of some limitations including being limited to only three cutaneous, respiratory and ocular complications and an overall look on carcinogenesis MG and cancer prevalence in MG exposure victims. It would have been more appropriate if carcinogenic effect of the agent and other complications including quality of life and psychological disorders of these subjects were studied in more details. Therefore, the authors suggest similar studies in the aforementioned fields to be designed and conducted. So further epidemiological and experimental studies are necessary to determine the target sites and nature of the carcinogenic risk from MG exposure to humans.

\section{Acknowledgements}

This study was supported by Baqiyatallah University of Medical Sciences and Tabriz University of Medical Sciences.

\section{References}

Abbas F (1984). Report of the specialists appointed by the Secretary-General of the United Nations to investigate allegations by the Islamic Republic of Iran concerning the use of chemical weapons. Archives belges $=$ Belgisch archief, 302.

Agin K, Broumand G (2004). Epidemiological relationship with late complications of sulfur mustard gas on 500 Iranian chemically injured after 15 years. J Army Univ Med Sci I.R.Iran, 2, 269-73.

Amirnia M, Babaie-ghazani A, Fakhrjou A, et al (2014). Immunohistochemical study of cyclooxygenase-2 in skin tumors. J Dermatolog Treatment, 25, 380-7.

Balali-Mood M, Hefazi M, Mahmoudi M, et al (2005). Longterm complications of sulphur mustard poisoning in severely intoxicated Iranian veterans. Fundamental \& Clinical Pharmacology, 19, 713-21.

Barar J, Omidi Y (2012). Translational approaches towards cancer gene therapy: hurdles and hopes. BioImpacts: BI, 


\section{2, 127.}

Bhavana SM, Lakshmi CR (2014). Oral Oncoprevention by Phytochemicals-A Systematic Review Disclosing the Therapeutic Dilemma. Advanced Pharmaceutical Bulletin, 4, 413.

Chatterjee D, Mukherjee S, Smith MG, et al (2003). Signal transduction events in lung injury induced by 2 chloroethyl ethyl sulfide, a mustard analog. J Biochemical and Molecular Toxicol, 17, 114-21.

Doi M, Hattori N, Yokoyama A, et al (2011). Effect of mustard gas exposure on incidence of lung cancer: a longitudinal study. Am J Epidemiol, 173, 659-66.

Easton D, Peto J, Doll R (1988). Cancers of the respiratory tract in mustard gas workers. Br J Industrial Med, 45, 652-9.

Eisenmenger W, Drasch G, Clarmann Mv, et al (1991). Clinical and morphological findings on mustard gas [bis (2-chloroethyl) sulfide] poisoning. J Forensic Sci, 1688-98.

Emad A, Rezaian GR (1997). The diversity of the effects of sulfur mustard gas inhalation on respiratory system 10 years after a single, heavy exposure analysis of 197 cases. CHEST $J, 112,734-8$.

Emadi S, Mortazavi M, Mortazavi H (2008). Late cutaneous manifestations 14 to 20 years after wartime exposure to sulfur mustard gas: A long-term investigation. Archives of Dermatology, 144, 1059-61.

Fraumeni Jr J (1975). Respiratory carcinogenesis: an epidemiologic appraisal. J National Cancer Institute, 55, 1039.

Geraci MJ (2008). Mustard gas: imminent danger or eminent threat? Ann Pharmacotherapy, 42, 237-46.

Ghabili K, Agutter PS, Ghanei M, et al (2010). Mustard gas toxicity: the acute and chronic pathological effects. J Applied Toxicol, 30, 627-43.

Ghabili K, Shoja MM, Golzari SE, et al (2013). Mustard gas keratitis: a common misnomer. Cornea, 32, 382-3.

Ghaneei M, Alavian S, Nasiri M, et al (2007). Alanin aminotransferase activity in veterans exposed to sulfur mustard. Iranian Journal of Endocrinology and Metabolism (IJEM).

Ghanei M, Vosoghi AA (2002). An epidemiologic study to screen for chronic myelocytic leukemia in war victims exposed to mustard gas. Environmental health perspectives, 110, 519.

Gharemohammadlou R, Emad A (2013). Evaluating Installation of Local Steroids in Lung Volumes and Airway Resistance in Bronchiectasis Due to Mustard. Med J Tabriz University Med Sci Health Serv, 35, 72-7

GhasemiBroumand M, Amiri Z (2008). Layed ocular complications of mustard gas in chemical injured 500 people. J Rehab, 8, 67-74.

GhasemiBroumand M, Aslani J, Emadi N, et al (2006). Frequency of late ocular, dermatological and respiratory complications due to exposure of mustard gas in Sardasht 2004. Pejouhandeh, 11, 9-15.

GhasemiBroumand M, Karami R, Pourfarzam S, et al (2007). Late complications of ocular, respiratory, dermatological and psychiatric (PTSD) caused by chemical agents. Daneshvar Med, 14, 81-92.

Gilasi H, Holakouie Naieni K, Zafarghandi M, et al (2006). Relationship between mustard gas and cancer in Iranian soldiers of imposed war in Isfahan Province: A Pilot Study. $J$ School of Public Health and Institute of Public Health Research, 4, 15-23.

Heston W (1953). Occurrence of tumors in mice injected subcutaneously with sulfur mustard and nitrogen mustard. J National Cancer Institute, 14, 131-40.

Jabbari H, Alikhah H, Sahebkaram Alamdari N, et al (2012). Developing the Use of Quality Indicators in Sterilization
Practices. Iranian J Public Health, 41, 64-9.

Karalliedde L, Wheeler H, Maclehose R, et al (2000). Possible immediate and long-term health effects following exposure to chemical warfare agents. Public Health, 114, 238-48.

Kehe K, Szinicz L (2005). Medical aspects of sulphur mustard poisoning. Toxicology, 214, 198-209.

Le H, Knudsen S (2006). Exposure to a first world war blistering agent. Emergency Medicine Journal, 23, 296-9.

Manning K, Skegg D, Stell P, et al (1981). Cancer of the larynx and other occupational hazards of mustard gas workers. Clinical Otolaryngology \& Allied Sciences, 6, 165-70.

Marzban S (1986). Treatment of Iran in mustard gas injuries. University lahadoftrnn, 1, 1-25.

McClintock SD, Till GO, Smith MG, et al (2002). Protection from half mustard gas induced acute lung injury in the rat. J Applied Toxicology, 22, 257-62.

Mirsadraee M, Attaran D, Boskabady M, et al (2005). Airway hyperresponsiveness to methacholine in chemical warfare victims. Respiration, 72, 523-8.

Mortazavi H, Baharvand M, Mehdipour M (2014). Oral potentially malignant disorders: an overview of more than 20 entities. J Dental Res, Dental Clinics, Dental Prospects, 8,6 .

Najafi A, Masoudi-Nejad A, Imani Fooladi AA, et al (2014). Microarray gene expression analysis of the human airway in patients exposed to sulfur mustard. J Receptors and Signal Transduction, 34, 283-9.

Namazi S, Niknahad H, Razmkhah H (2009). Long-term complications of sulphur mustard poisoning in intoxicated Iranian veterans. J Med Toxicol, 5, 191-5.

Naraghi ZS, Mansouri P, Mortazavi M (2005). A clinicopathological study on acute cutaneous lesions induced by sulfur mustard gas (yperite). European Journal of Dermatology, 15, 140-5.

Nishimoto Y, Yamakido M, Shigenobu T, et al (1983). Long-term observation of poison gas workers with special reference to respiratory cancers. J UOEH, 5, 89-94.

Norman JE (1975). Lung cancer mortality in World War I veterans with mustard-gas injury: 1919-1965. J National Cancer Institute, 54, 311-7.

Program NT (2011). NTP 12th Report on Carcinogens. Report on carcinogens: carcinogen profiles/US Dept. of Health and Human Services, Public Health Service, National Toxicology Program, 12, 3 .

Ray R, Keyser B, Benton B, et al (2008). Sulfur mustard induces apoptosis in cultured normal human airway epithelial cells: evidence of a dominant caspase-8-mediated pathway and differential cellular responses*. Drug and chemical toxicology, 31, 137-48.

Riazi A, Jadidi K, Naderi M, et al (2004). Refractive errors in victims of chemical mustard as a delayed complication. $J$ Mil Med, 6, 13-8.

Safarinejad M (2001). Testicular effect of mustard gas. Urology, $\mathbf{5 8}, 90-4$.

Saleh P, Bastani P, Piri R, et al (2013). Antimicrobial prophylaxis for surgical site infections in surgical wards in northwest Iran. Life Sci J, 10, 1977-81.

Sasser L, Miller R, Kalkwarf D, et al (1996). Subchronic toxicity evaluation of sulfur mustard in rats. Journal of Applied Toxicology, 16, 5-13.

Security SS-cotBHSttMoH (2011). Report on mustard gas experiments (Glasgow and London). Homeopathy, 100, 27-35.

Shigenobu T (1980). Occupational cancer of the lungs--cancer of the respiratory tract among workers manufacturing poisonous gases. Nihon Kyōbu Shikkan Gakkai zasshi, 18, 880.

Sokouti M, Golzari SE, Pezeshkian M, et al (2013). The role of 

esophagogastric anastomotic technique in decreasingbenign stricture formation in the surgery of esophageal carcinoma. J Cardiovascular Thoracic Res, 5, 11 .

Soroush MR, Ghanei M, Assari S, et al (2009). Urogenital history in veterans exposed to high-dose sulfur mustard: a preliminary study of self-reported data. Urology journal, 6, 114-9.

Stuart JA, Ursano RJ, Fullerton CS, et al (2003). Belief in exposure to terrorist agents: reported exposure to nerve or mustard gas by Gulf War veterans. The Journal of nervous and mental disease, 191, 431-6.

Tokuoka S, Hayashi Y, Inai K, et al (1986). Early cancer and related lesions in the bronchial epithelium in former workers of mustard gas factory. Pathology International, 36, 533-42.

Wada S, Miyanishi M, Nishimoto Y, et al (1968). Mustard gas as a cause of respiratory neoplasia in man. The Lancet, 291, 1161-3.

Zafarghandi MR, Soroush MR, Mahmoodi M, et al (2013). Incidence of cancer in Iranian sulfur mustard exposed veterans: a long-term follow-up cohort study. Cancer Causes \& Control, 24, 99-105.

Zandieh T, Marzban S, Hassiri G, et al (1990). Evaluation of cell-mediated immunity in mustard gas injuries. Medical Journal of The Islamic Republic of Iran (MJIRI), 4, 257-60.

Zarchi K, Akbar A, Naieni KH (2004). Long-term pulmonary complications in combatants exposed to mustard gas: a historical cohort study. International Journal of Epidemiology, 33, 579-81.

Zojaji R, Balali-Mood M, Mirzadeh M, et al (2009). Delayed head and neck complications of sulphur mustard poisoning in Iranian veterans. The Journal of Laryngology \& Otology, 123, 1150-4. 